\title{
Reversible delayed lexical choice in a bidirectional framework
}

\author{
Graham Wilcock* \\ Centre for Computational Linguistics \\ University of Manchester Institute \\ of Science and Technology \\ PO Box 88, Manchester M60 1QD \\ United Kingdom \\ graham@ccl.umist . ac .uk
}

\author{
Yuji Matsumoto \\ Graduate School of Information Science \\ Nara Institute of Science and Technology \\ 8916-5 Takayama, Ikoma, Nara 630-01 \\ Japan \\ matsu@is.aist-nara.ac.jp
}

\begin{abstract}
We describe a bidirectional framework for natural language parsing and generation, using a typed feature formalism and an HPSG-based grammar with a parser and generator derived from parallel processing algorithms. We present an approach to delayed lexical choice in generation, based on subsurnption within the sort hicrarchy, using a lexicon of underinstantiated signs which are derived from the normal lexicon by lexical rules. We then show how delayed lexical choice can be used in parsing, so that some types of ill-formed inputs can bo parsed, but wellformed outputs are generated, using tho same shared linguistic information.
\end{abstract}

\section{A bidirectional framework}

In our bidirectional framework for a natural language engine, the linguistic descriptions in the grammar and lexicon are shared resources which are used, in appropriately compiled forms, for both parsing and generation. Like the Core Language Engine (CIE) (Alshawi, 1992), we use a unification-based phrase structure grammar, a logical form representation, a bottom-up chart, parser and a semantic head-driven generation algorithm. However, we differ from CLE by exploiting recent developments in processing algorithms and in linguistic representation formalisms. Wo will clarify the similarities and differences at the relevant points.

\subsection{Processing algorithms}

The SAX parser is a concurrent SICStus Prolog implementation of the PAX parallel parsing algorithm (Matsumoto and Sugimura, 1987; Matsumoto et al., 1994). Like the earlier BUP parser to which the CIE parser was closely related, SAX uses partial execution to compile the grammar for efficient bottom-up parsing. However, instead of

\footnotetext{
${ }^{*}$ Visiting researcher of Information Systems Prod uct Development Laboratories, Sharp Corporation.
}

building the chart by asserting edges into the Prolog database, SAX implements the chart by creating concurrent processes. For compiled rather than interpreted Prolog systems, this is a highly efficient form of chart parsing, even on sequential machines. The terminal and non-terminal symbols of the grammar are realized as processes which communicate via streams to build larger structures. A meta-process monitors the streams and controls the whole parsing process.

The SGX generator is a concurrent SICStus Prolog implementation of the BCG parallel bidirectional chart generation algorithm (Haruno ot al., 1993; Den, 1994). SGX uses partial execution to compile the grammar for efficient generation. Like the CLE genorator, SGX follows the semantic head-driven (SHD) generation algorithm (Shieber et al., 1990) to ensure efficient ordering of the generation process. However, SGX also implements the suggestion of Shieber et.al. that backtracking and recomputation of results should be avoided by using a chart, as in chart parsing. Like SAX, SGX implements the chart by concurrent processes and communication streams monitored by a meta-process.

SAX and SGX accept definite clause grammars, with specific requirements to eliminate nondeterminism. Prolog code can be added to DCG rules as extra conditions, but the extra conditions must. be deterministic. Empty categories are not supported in normal processing, ${ }^{1}$ and SGX does not allow non-chain rules with uninstantiated logical forms.

\subsection{Grammar formalism}

Large DCG-based grammars typically have many rules, many categories, and many arguments per category. Such grammars could be efficiently procossed by SAX and SGX, but are difficult to de-

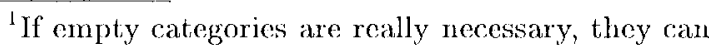
be handled in the concurrent processing system via a meta-process. This approach is described in (Imaichi and Matsumoto, 1995) for ill-formed inputs. However, we eliminate traces by lexical rule, and welcome the proposals of (Sag, 1995) for eliminating all empty categories from HPSG.
} 
velop and debug. CLI addressed this problem by adopting GPSG grammatical thoory and expressing linguistic descriptions as feature structures, but the CIs grammar still had many arguments per category and many rules. Wo adopt HIPSG grammatical theory (Pollard and Sag, 1994) and express linguistic descriptions in a typod feature formalism, but we still implement the grammar in DCG form.

Since HPSG collects all foatures into a structured sign, the many DCG arguments are replaced by a single HPSG sign. As HISSG genoralizes from category-based rules (for S, NP, etc.) to schemas for phrasal signs, the many DCG rules are replaced by a fow generalized rules. Wo specify a separate logical form (LF) for generation, as in (Shieber ot al., 1990). On DCG catcgories therefore have the format word(Sign)/LF and phrase(Sign)/LF. ${ }^{2}$

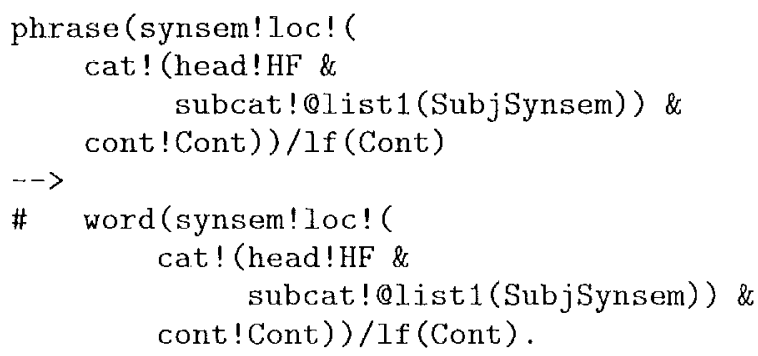

Figure 1: 'The source form of a grammar rule

Figure 1 shows the source form of a simplified version of IIPSG Schema 2 with zero complement daughters. Qlist 1 is a template which oxpands to a list with one member. The \# symbol is required by SGX to identify the semantic hoad of a chain rule for SHD generation.

\subsection{Grammar compilation}

The ProFIT system (Erbach, 1995) is an extension of Prolog which supports a typed foature formalism with multiple inheritance. CLE used a sort hierarchy only for somantic solectional rostrictions. HPSG uses a sort hicrarchy also for syntactic restrictions, and exploits multiple inheritance for lexicon organization.

\footnotetext{
${ }^{2}$ In this paper we follow (Shicber et al., 1990) and (Pollard and Sag, 1994) in equating logical form with semantic content. A separate logical form is therefore redundant, as the content feature could be used to control SHD generation. However, logical form may need to include other information, such as unscoped quantifiers (IIPSG qstore), presuppositions (IIPSG context), pragmatic and discourse factors (in fact Shieber et al. include mood operators). An important consequence of this is that a semantic head in the sense of the HPSG Content Principle is not necessarily a semantic head in the sense of the SHD generation algorithm.
}

ProHtT compiles the typed feature formalism into Prolog terms, which can be used with any appropriate parser or generator. We therefore use ProlIT in order to combine IIPSG grammar with the SAX parser and the SGX generator, by compiling the grammar in two separate stages.

In the first, stage, the typed feature structures in the I) CG rules are compiled by ProIT' into efficient Prolog terms. Like CIA, Prol'IT compiles foature structures into terms, so that relatively slow unification of feature structures is roplaced by relatively fast unification of terms. Also like CLE, Prol:T uses the technicue of (Mellish, 1988) for compiling finite domains such as index agreement into boolean vectors for fast unification.

In the second stage, the resulting I)CG containing only Prolog terms is compiled separately by the SAX translator for parsing and by the SGX translator for generation. Grammar rules can be: labelled to be compiled only by SAX or only by SGX, so that parsing could for example use some rules with wider coverage than others used in gencration, while sharing most of the grammar.

Like the carlier BUP parser, the SAX translator uses partial execution to produce efficient codo for bottom-up chart parsing. 'The SGX translator' compiles tables of chain rules and also uses partial execution to produce efliciont code for SHD chart generation.

\subsection{Lexicon compilation}

We do not compile the lexicon ofl-line into a static list of signs. Instead, the existence of a lexical sign is proved on-line by lexical inference rules. Wo specify a morphological lexicon intcrface

morph_lex(Form, Cat, [LF, Sign])

where Form is a specific morphological form, and Sign is a typed feature structure. A lexical inforcnce rule is shown in simplified form in Figuse 2. In ProflT, sorts are written as <sort, and fealures as feature!value.

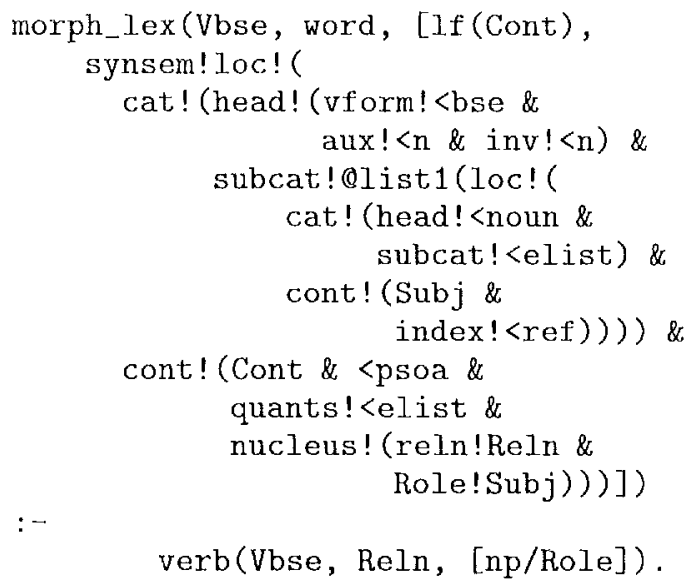

Figure 2: $A$ morph_lex rule for a verb base form 
We use lexical inference rules to derive full HPSG lexical signs from a database of simple Prolog clauses. Such rules can partially re-use available non-HPSG lexical information. The example assumes a lexical entry such as

verb (walk, walk1, [np/agent])

specifying a verb with base form walk and sense walk1, which subcategorizes for a noun phrase subject assigned to a thematic role agent.

We also use rules like normal HPSG lexical rules, to derive new signs from other lexical signs for morphological derivations, complement extraction and so on. We have no automatic defaults, so these rules must be written carefully. The simplified example in Figure 3 instantiates nominative and 3rd singular in the first subcat item, and copies the rest of subcat by unification.

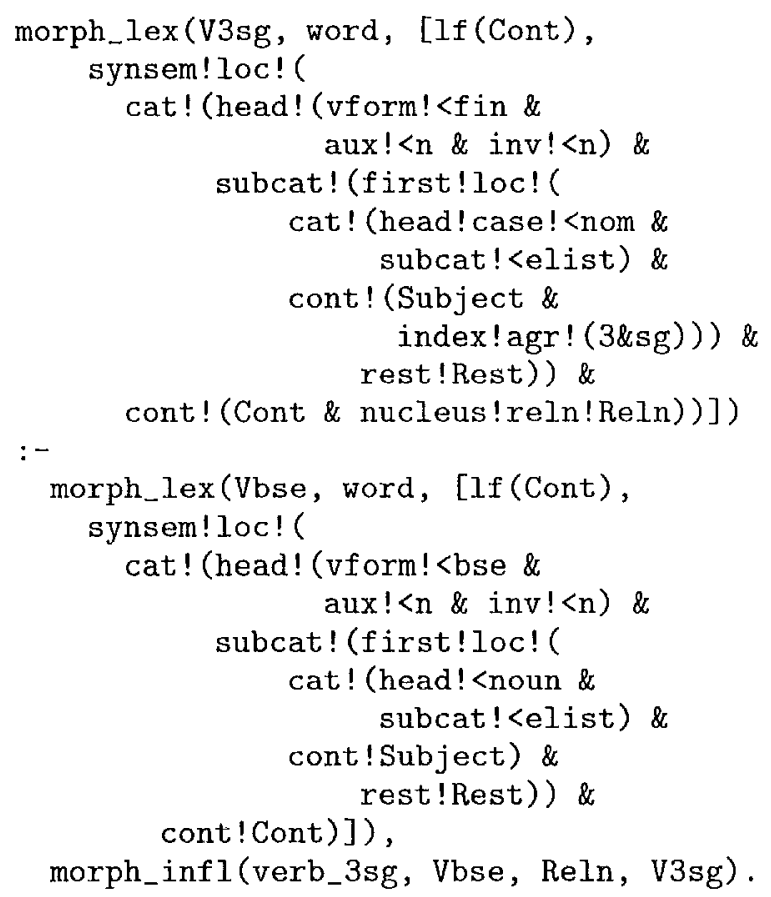

Figure 3: Lexical rule for 3rd singular verb form

The typed feature structures in the lexical rules are compiled by ProFIT into Prolog terms. The resulting rules are then compiled by SICStus Pro$\log$, together with the database of simple lexical entries.

\section{Delayed lexical choice}

Delayed lexical choice is an established technique in natural language generation. When a backtracking algorithm is combined with a lexicon of morphological forms, there is considerable nondeterminism during syntactic generation, because features required for a deterministic choice of morphological form are not yet instantiated. With delayed lexical choice, a lexicon of stems is used dur- ing syntactic generation, and the choice of morphological form is delayed to a postprocess. Instead of producing a string of word forms, syntactic generation produces a string of lexical items. The morphological postprocess converts the lexical items to final lexical forms, when all required syntactic features have become instantiated.

\subsection{Monotonicity}

Describing the implementation of delayed lexical choice in the MiMo2 system, Shieber et al. (1990) pointed out that only monotonic rules (which further instantiate the feature structure of a lexical item but do not change it) can be delayed. For example, the choice of singular or plural verb form can be delayed until after the subject has been generated, by performing syntactic gencration with a lexical item based on the verb stem, which does not specify singular or plural. By contrast, a lexical rule for passivization which changes the order of items on the subcat list is nonmonotonic. Both the active and the passive variants must be made available as distinct lexical items during syntactic generation. ${ }^{3}$

In an inheritance-bascd typed feature formalism, monotonicity is built into the subsumption relation in the sort hierarchy. A sort subsumes its subsorts, which may further instantiate its features, but cannot change them. Wo exploit the monotonicity of subsumption in the sort hierarchy in our implementation of delayed lexical choice.

\subsection{Syntactic-semantic lexicon}

In place of the MiMo2 lexicon of stems, we specify a syntactic-semantic lexicon interface

synsem_lex(Lex, Cat, [LF, Sign])

where Lex has no significance for generation. Entries in the syntactic-semantic lexicon are derived by a small number of lexical rules from entries in the morphological lexicon. Like the morph_lex rules, the synsem_lex rules are compiled first by ProFIT and then by SICStus Prolog.

To implement delayed lcxical choice, we use the synsem_lex interface during syntactic generation, and then use the morph lex interface in the morphological postiprocess. We must therefore ensure that the delayed morph_lex rules will be monotonic. We do that by ensuring that the synsem.lex entries subsume the morph lex entries from which they are derived.

Figure 4 shows a simplified form of a synsem.lex rule for verbs. The rule derives the synsem_lex entry from the morph_lex base form entry, in which vform has a value of sort <bse. The subcat of the synsem.lex entry is unified with the subcat of the

\footnotetext{
${ }^{3}$ We currently make such variants available via alternative forms of the lexicon access rule (Section 2.3). This could be improved by using constraints to enable subcat-changing rules to be delayed (van Noord and Bouma, 1994; Meurers and Minnen, 1995).
} 
morph_lex entry, so that the synsem_lex cntry subcategorizes for the appropriate syntiactic complements. The morph_lex base form cntry is used so that the agreoment features of the subject will not: be restricted. The content values are also unified, so that the synsem_lex entry includes the appropriate semantic roles. However, the head features are not unified. The synsem lex vorm has a value of sort < vform, which is the immediate supersort. of the morph_lex vform sort <bse. Instead of full unification, the synsem lex head features subsume those of the morph_lex entry.

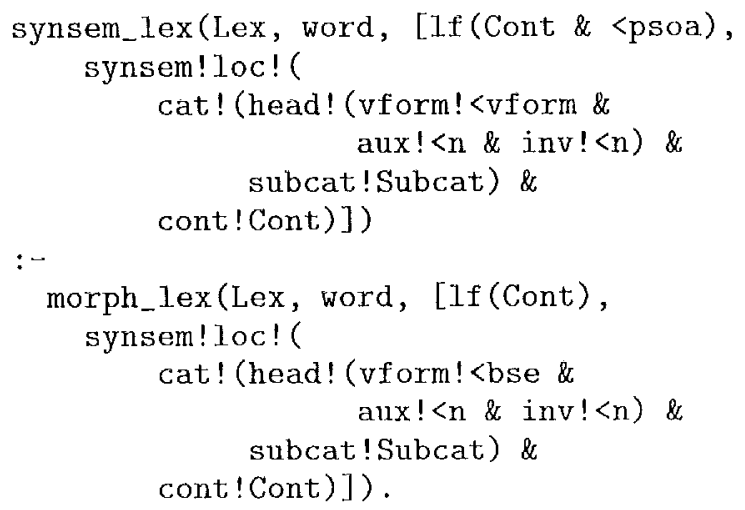

Figure 4: A synsem_lex rule for verbs

\subsection{Grammar-lexicon interface}

In DCG-based systems, the interface between the grammar and the lexicon can often be specificd by a DCG rule which accesses the lexicon by moans of an extra condition. In our framework, such a rule might be:

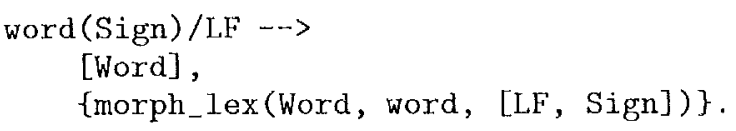

However, since our concurrent processing algorithms require extra conditions to be deterministic, such a rule would find only one lexical entry (the first unifiable one), which would depend on the order of lexical rules and lexical entries.

For parsing this is not a problem because, like CLE, we use a morphological preprocess which performs lexicon access, building a morphemo lattice to be passed to the syntactic parsing process. Lexicon access is therefore separated from the parsing algorithm, and is not required to be deterministic.

For generation with delayed lexical choice, we use a modified form of the rule:

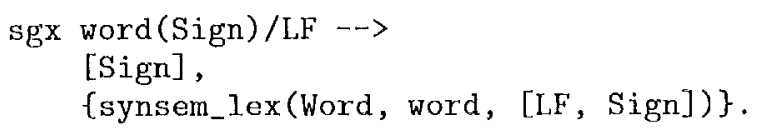

The label 'sgx' shows that the rule is to be compiled only by SGX, not by SAX. It differs from the previous rule not only by accessing the syntacticsemantic lexicon instead of the morphological lexicon, but also by specifying that the lexical item is [Sign] instear of [Word]. That is, the output of syntactic gencration is a string of HPSG signs.

\subsection{Semantic head-driven generation}

When syntactic generation begins, the SHD algorithrn uses chain rules (like the rule in Figure 1) to identify the pivot, the semantic head of the sentence. 'I'he synsem_lex entry for the pivot is then accessed by the extra condition in the DCG rule above.

Since the synsem_lex entry for verbs (Figure 4) does not specify subject agrecment or vform subtype, but does specify subcategorization and semantic roles, it can be used equally well as the scmantic head to drive syntactic generation of, saly, a 3rd-singular finite clause or an infinitival complement. Since a single entry can be used in this way, the extra condition can be deterministic, as required.

If the verb is the head of an infinitival complement, its vform becomes instantiated to <bse from subcategorization by the auxiliary to. If the verb is the hear of the main clause, its vform becomes instantiated to $<$ fin (finite) by a rule for grammatical unitis in our grammar. ${ }^{4}$

After syntactic generation, the string of IFPG signs is converted to a string of word forms by a morphological postprocess, which unifies the signs with entries in the morphological lexicon. As the signs are fully instantiated during syntactic geueration, this postprocess is also deterministic.

\section{Reversible delayed lexical choice}

Most forms of robust parsing are based on constraint rolaxation. Our approach to delayed lexical choice is based on using less instantiated signs from the syntactic-somantic lexicon, rather than the more instantiated signs from the morphological lexicon. This can be viewed as equivalent to constraint relaxation. It therefore secrns reasonable to consider reversing the approach, using delayed lexical choice for parsing.

Constraint relaxation in parsing typically has a two-pass approach. Strict parsing is attempted with normal grammar rules and the normal parsing algorithm. If strict parsing fails to produce a parse, relaxed parsing is attempted, using a modified algorithm or modified grammar rules. With a lexicalist grammar like HPSG it seems more appropriate to use modified lexical rules, as in our syntactic-semantic lexicon.

\footnotetext{
${ }^{4}$ In addition to the HPSG categories word and phrase, we have a category gram-unit for grammatical units which can be uttered independently. These include finite sentences, accusative NPs, and others The rule for gram_unit thus instantiates certain features which are required for well-formed generation.
} 
However, in our approach to delaycd lexical choice we do not start with strict constraints and then relax them. On the contrary, we start with relaxed constraints from less instantiated signs and then further instantiate the signs as other constraints become available. Our approach is thercfore incremental description refinement (Mellish, 1988) rather than constraint relaxation.

\subsection{Parsing and generation with delay}

When the syntactic-semantic lexicon is used for generation, the logical form is the retrieval key, and the name of the lexeme is irrelevant. In the interface synsem_lex(Lex, word, [LF, Sign]), the variable Lex does not need to be unificd with the name of the morpherne in the morph_lex entry, and could be given another value, such as "verb". However, if we use the syntactic-semantic lexicon for parsing, the value of this variable will be the retrieval koy. If the value is taken directly from the words of the input string, it will not necessarily unify with the name of the morpheme in the morph_lex entry.

In the case of verbs (Figure 4), where the input word may be an inflected form but the synsem.lex entry uses the morph lex entry for the base form, we must first use the morphological preprocess to obtain the "root" form of the word, which is the same as the base form. We then use the root form instead of the input form as the retrieval key. In the case of pronouns, which take different forms according to case and reflexivity but have no natural root form, the input form is used directly as the retrieval key (Section 3.2).

Since the synsem_lex entry for verbs in Figure 4 does not restrict subject agreement, an illformed input with incorrect subject-verb agreement is parsed in exactly the same way as a wellformed input. The'subject agreement in the verb's sign remains uninstantiated until the subject and the verb phrase are combined by Schema 1, when the agreement features are instantiated to those of the subject. So "she swim" is accepted, but only "she swims" is generated in a finite clause. The synsem.lex entry in Figure 4 also does not restrict vform, which remains uninstantiated until the verb phrase is combined into a larger phrase. So "she can swimming" is accepted, but only "she can swim" is generated, since "can" subcategorizes for a VP with vform of sort <bse.

\subsection{Experimenting with delay}

Of course, different specifications in the rules for the syntactic-scmantic lexicon produce different effects. In the synsem_lex entry for pronouns in Figure 5, instead of unifying the head fcature case with the morph.lex entry, the head is specified only as sort <noun, leaving the case unspecified. There are distinct morph_lex entries for nominative and accusative forms of personal pronouns, but it is irrelevant which one happens to be found when the rule is executed, because the rule does not unify the head features which include case. So the synsem_lex cntry can be used deterministically for syntactic gencration, leaving the case to be instantiated from subcategorization by a verb or preposition.

In parsing, the effect of this form of the rule is that the case of an input pronoun is ignored. Whether this is good or bad depends on both the language and the level of relaxation desired. This form of the rule would clearly be unsuitable for free word ordor languages, but seems useful for English, accepting "for you and I" but generating "for you and me".

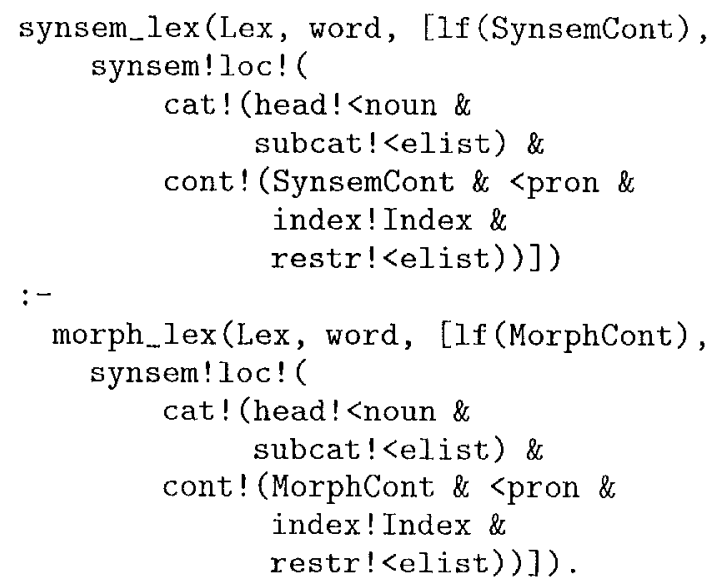

Figure 5: A synsem_lex rule for pronouns

In Figure 5, the synsem_lex content value is not unified with the morph lex content value. Only the index values are unified, including the gender, number and person features essential for pronouns (the restr values are ompty lists). The contant values are constrained only to be of sort <pron (pronominal). In the sort hierarchy, <pron has subsorts <ana (anaphoric) and <ppro (personalpronominal), and <ana has its own subsorts < refl. (reflexive) and <recp (rociprocal). HPSG binding theory is based on these sortal distinctions, which are part; of the content value.

Again, there are distinct morph_lex entries for reflexive and personal-pronominal forms, but it is irrelevant which one happens to be found when the rule is executed, because the rule does not unify the content values. Therefore the synsem_lex entry can be used deterministically for syntactic generation before the sort becomes instantiated to <ana or <ppro by the binding principles.

The effect of this form of the rule is to relax the binding constraints in parsing, accepting "I saw me" but generating "I saw myself". Of course tho distinction between "They saw themselves" (coindexed) and "They saw them" (contra-indexed) is also lost in parsing with this version. The bind- 
ing constraints can be re-instated simply by unifying the content values in the rule, but the above version is not necessarily bad, for example in parsing non-native English. The rule could bo improved by having alternative forms which distinguish 3rd and non-3rd person.

\section{Conclusion}

Our framework secks to combine the elegance of a typed feature formalism and IIPSG syntactic theory with efficient processing. The ProFIT' system gives an efficient encoding of typed feature structures. The SAX and SGX systems use an efficient, chart implementation, and their concurrent. processing algorithms give firther motivation for eliminating empty categories and reducing nondeterminism.

We have addressed the issue of bidirectional use of shared linguistic descriptions, rather than robust, parsing. However, the idea of using delayed lexical choice in reverse makes it possible, without. modifying the parsing and generation algorithms, to parse certain types of ill-formed inputs and to generate corresponding well-formed outputs, using the same shared linguistic descriptions.

\section{Acknowledgements}

The first anthor would like to thank Mr Hitoshi Suzuki (Sharp Corporation) and Prof Jun-ichi Tsujii (UMIST) for making this work possible. We also thank Dr Kristiina Jokinen (NAIST) and the anonymous reviewers for valuable commonts.

\section{References}

Hiyan Alshawi, editor. 1992. The Core Language Engine. The MIT Press.

Yasuharu Den. 1994. Generalized chart algorithm: an efficient procedure for cost-based abduction. In 32nd Annual Mecting of the Association for Computational Linguistics, pages 218-225. Association for Computational Linguistics.

Gregor Frbach. 1995. ProFIT: Prolog with Features, Inheritance, and Templates. In Seventh Conference of the European Chapter of the Association for Computational Linguistics. Association for Computational Linguistics.

Masahiko Haruno, Yasuharu Den, Yuji Matsumoto, and Makoto Nagao. 1993. Bidirectional chart generation of natural language texts. In Proceedings of the Lleventh National Conference on Artificial Intelligence, pages 350-356. AAAI Press/The MIT Press.

Osamı Imaichi and Yuji Matsumoto. 1995. Integration of syntactic, semantic and contextual information in processing grammatically ill-formed inputs. In Procedings of the Fourtecnlh International Joint Conference on Artificial Intelligence, pages 143540.

Yuji Matsumoto and Ryoichi Sugimmra. 1987. $\Lambda$ parsing system based on logic programming. In Proceedings of the T'enth International Joint Conference on Artificial Intelligence, volumo: 2, pages 6714.

Yuji Matsumoto, Yasuharu 1)en, and Takehito Utsuro, 1994. Koubun kaiseki shisutemu $S A X$, shiyou selsumeisho (Parsing system SAX Manual) version 2.1. Nara Institute of Scicnce and Technology.

Christopher S. Mollish. 1988. Implementing systomic classification by unification. Computational Linguistic, 14(1):40 51.

W. Detmar Meners and Guido Minnen. 1995. A computational treatment of IIPSG lexical rules as covariation in lexical entries. In Proceedings of the Fifth International Workshop on Natural Language Understanding and Logic I'rogramming, Lisbon, Portugal.

Carl Pollard and Ivan Sag. 1994. Head-driven Phrase Structure Grammar. University Press, Chicago.

Ivan Sag. 1995. English rolative clause constructions. Unpublished manuscript.

Stuart M. Shieber, Gertjan van Noord, Fernando C.N. Pereira, and Robert C. Moore. 1990. Semantic: head-driven generation. Computational Linguistics, 16(1):30-42.

Gertjan van Noord and Gosse Bouma. 1994. Adjuncts and the processing of lexical rules. In 15th International Conference on Computational Linguistics. Association for Computational Tinguistics. 